\title{
Synthesis and characterization of TEMPO-oxidized peptide- cellulose conjugate biosensors for detecting human neutrophil elastase
}

\author{
Robert T. Mackin • Krystal R. Fontenot $\cdot$ J. Vincent Edwards $\mathbb{\bullet} \cdot$ \\ Nicolette T. Prevost • Casey Grimm • Brian D. Condon - Falk Liebner • \\ Jacobs H. Jordan • Michael W. Easson • Alfred D. French
}

Received: 24 June 2021 / Accepted: 1 December 2021/Published online: 9 January 2022

This is a U.S. government work and not under copyright protection in the U.S.; foreign copyright protection may apply 2021

\begin{abstract}
Here we describe the synthesis and characterization of a peptide-cellulose conjugate biosensor based on TEMPO-oxidized nanofibrillated cellulose (tNFC) for detecting elevated levels of human neutrophil elastase (HNE) in chronic wounds. The fluorescent peptide HNE substrate constructed from n-succinyl-Ala-Pro-Ala-7-amino-4-methyl-coumarin was attached to the TEMPO-oxidized cellulose surface via polyethylene glycol linker. The characterization of the biosensor conjugate shows a high degree of peptide incorporation onto the surface with the degree of substitution of 0.057 . The relatively small crystallite size of $26.0 \AA$ compared to other cellulose- and nanocellulose-based materials leads to a large specific surface area which can promote access of HNE to the enzyme substrates due to decreased steric interactions. Likewise, the porosity for tNFC was found to be higher than all other samples, including the nanocellulosic aerogel, lending to its hydrogel-like nature.
\end{abstract}

R. T. Mackin · K. R. Fontenot · J. V. Edwards ( $\square)$.

N. T. Prevost - C. Grimm · B. D. Condon ·

J. H. Jordan · M. W. Easson - A. D. French

United States Department of Agriculture, Agricultural

Research Service, Southern Regional Research Center

(USDA-ARS-SRRC), New Orleans, LA 70124, USA

e-mail: vince.edwards@ars.usda.gov

F. Liebner

Division of Chemistry of Renewable Resources,

University of Natural Resources and Life Sciences

Vienna, Konrad-Lorenz-Straße 24,

3430 Tulln an der Donau, Austria
The properties of tNFC were compared to other cellulose-based materials. The volume of each crystallite and volume ratio to the largest sample was calculated. tNFC was found to occupy the smallest space resulting in high amounts of sensors per crystallite unit volume. With a small crystallite volume and large number of sensors, the tNFC peptide-cellulose conjugate biosensor could provide a more sensitive system and is a good candidate for point of care diagnostic devices for detecting elevated protease levels in humans.

Keywords Peptides - TEMPO-oxidized nanofibrillated cellulose $\cdot$ Human neutrophil elastase . Biosensors

\section{Introduction}

Biosensors are devices that employ biochemical interactions to monitor the presence of specific compounds at minimal concentrations, either directly or indirectly, based on the system design. These sensors are used in a variety of fields ranging from environmental testing to detecting pathogens in the human body (Mohanty et al. 2006; Soper et al. 2016). Because these sensors operate on the nanoscale, they allow for in vivo use and pointed evaluation of an individual's symptoms (Jurado-Sánchez 2018; Thévenot et al. 1999). Biosensor technologies have the 
potential to transform the healthcare industry through non-invasive assessment of disease and related health issues such as sepsis (Kumar et al. 2013; Min et al. 2018). Over recent decades, much of the research into biosensor systems has focused on the growing use of point-of-care (POC) diagnostic devices that can be employed to rapidly assess the causes of chronic illnesses in patients, such as those associated with diabetes or related to being bedridden (Choi et al. 2011; Liu et al. 2012; Wang 2006). These devices are designed with a receptor-recognition component, which is activated via cellular or biochemical activity to generate the detected signal.

Nanomaterials with biocompatible properties and large specific surface area (SSA) offer increased sensitivity as transducer substrates for biosensors. Graphene-based nanomaterial devices are one such substrate and have received increased attention due to their biocompatibility and potential for surface modification (Chen et al. 2019; Huang et al. 2019). Until recently, graphene-based nanomaterials were limited as strictly 2-D materials, however 3-D materials have become available for sensor applications (Le Ferrand et al. 2020; Mao et al. 2020). However, these 3-D networks typically require a supporting porous scaffold, which must be removed, requiring complicated and time-consuming chemical processes.

Unlike graphene, nanocrystalline cellulose is a versatile material because of its hydrophilic nature, abundance of reactive hydroxy groups, and high SSA (Edwards et al. 2013a). Additionally, some forms of nanocellulose, namely aerogels and nanofibrillated cellulose, can take on a 3-D structure. This property affords the ability to utilize the material as a scaffold for regenerative tissue repair processes (Courtenay et al. 2018; Doench et al. 2019) and has been utilized for sensor applications (Blackwell 2006; Song et al. 2018), including electromechanical (Calahorra et al. 2018), plasmonic (Schlesinger et al. 2015) and biosensors (Zhang et al. 2010). Our previous research has illustrated the use of nanocrystalline cellulose as a POC diagnostic for serine proteases through the attachment of peptides to the transducer surface, known as a peptide-cellulose conjugate, detecting human neutrophil elastase (HNE) over a wide range of concentrations and using a variety of cellulose-based materials (Edwards et al. 2018a; Fontenot et al. 2016). Because the biosensor can be selectively tuned for specific pathogens, nanocellulose provides an effective foundation for these types of diagnostic systems with a high degree of sensitivity while interfacing with wound dressing motifs (Portela et al. 2019).

The variety of biosensor technologies for POC diagnostics provide electrical, optical, or mechanical signaling methods, and are detected by colorimetric, electrochemical, or fluorescent methods (Tamayo et al. 2013; Vasan et al. 2013). To promote functionality at varying degrees of sensitivity and resolution, detectors are integrated into matrices denoted as transducer surfaces. The interaction of the transducer surface with the sensor component and its effect on the signaling process is a function of both biocompatibility and spatiotemporal binding of the biomarker (Edwards et al. 2018a). However, despite improvements in detector technologies, studies on the interaction of biosensor with the transducer surface and its effect on analyte detection quality are limited. Here we report the synthesis and characterization of a fluorescent biosensor containing a tripeptide and PEG linker anchored to the transducer surface. The sensor is designed to examine the spatiotemporal interaction of a peptide-cellulose conjugate supported by TEMPOoxidized nanofibrillated cellulose (tNFC) and HNE. The material's physical properties are evaluated and compared to previous cellulose-based materials and biosensors to better understand which of these properties most affect detection sensitivity (Edwards et al. 2016a, 2016b, 2018a; Fontenot et al. 2016). The tNFC is found to contain a high degree of sensors per unit volume suggesting it would have increased sensitivity and be a valuable material to develop for POC diagnostics.

\section{Materials and methods}

\section{Materials}

$\mathrm{N}, \mathrm{N}$-dimethylformamide (DMF) and dichloromethane (DCM) were purchased from VWR. Ethylcyanoglyoxylate-2-oxime (Oxyma Pure), diisopropylcarbodiimide (DIC), N,N-diisopropylethylamine (DIPEA), 4-dimethylaminopyridine (DMAP), 1-Ethyl-3-[3dimethylaminopropyl] carbodiimide hydrochloride (EDC), N-hydroxysuccinimide (NHS) and sodium acetate (NaOAc) were purchased from MilliporeSigma. The 9-fluorenylmethyloxycarbonyl-amino- 
poly(ethylene glycol) ${ }_{3}$-amine (Fmoc-NH-PEG3-NH ${ }_{2}$ ) was purchased from Custom Synthesis, LLC (Anderson, SC). The elastase peptide substrate, n-succinylAla-Pro-Ala-(7-amino-4-methylcoumarin (Suc-APAAMC), was purchased from BACHEM. Human neutrophil elastase (HNE) was purchased from Athens Research Technologies. All chemicals were used as received without further purification. All general laboratory chemicals used were reagent grade. The 2,2,6,6-tetramethylpiperidine-1-oxyl (TEMPO)-oxidized cellulose nanofibrils (NFC), freeze-dried 98-99\% solids, was purchased from University of Maine Process Development Center.

\section{Methods}

\section{PEGylating of TEMPO-oxidized nanofibrillar cellulose ( $t N F C)$}

To the $0.5-1 \%$ suspension of tNFC, an aqueous solution of EDC $(0.1 \mathrm{M})$ and NHS $(0.4 \mathrm{M})$ in Milli-Qwater ( $\mathrm{pH} \sim 6.5$ ) was added with stirring for $20 \mathrm{~min}$ to activate the carboxyl groups and convert them to amine reactive esters. Then, Fmoc-amino-(tri-(ethylene glycol))-amine, (Fmoc-NH-PEG $-\mathrm{NH}_{2}$ ), dissolved in $10 \mathrm{mM} \mathrm{NaOAc}$ buffer at $\mathrm{pH} 5.0$ was added and stirred overnight. The excess reagents were deactivated with slightly basic sodium phosphate buffer and the products washed with deionized water. The amino-PEG nanocellulose conjugate was prepared for deprotection or stored for further use.

Deprotection of the amino-PEG nanocellulose conjugate was performed by soaking it in a $20 \%$ piperidine/DMF deblock solution for $15 \mathrm{~min}$ while sonicating. The deprotected amino-PEG-tNFC was washed with DMF. After that, the deprotected surfaces were prepared for peptide coupling or washed, air dried, and stored for further use.

\section{Immobilization of the peptide substrate}

To a $50 \mathrm{~mL}$ centrifuge tube with Oxyma Pure, DIC, and DMAP in milli molar equivalent ratio of (1:1:0.1), respectively, in DMF was added to the $\mathrm{NH}_{2}$-PEGtNFC stirring rapidly to thoroughly combine. To this solution, the respective peptide substrate (Suc-APAAMC) in DMF was added. The centrifuge tube was sonicated while maintaining the temperature at $25-30{ }^{\circ} \mathrm{C}$ for $3 \mathrm{~h}$ and then placed in the refrigerator overnight. The tNFC-PEG3-peptide conjugate was purified by washing thrice with DMF and then methanol using vacuum filtration or centrifuged and the supernatant decanted. During centrifugation, the sample was centrifuged at $6000 \mathrm{rpm}$ for $10 \mathrm{~min}$ to separate the peptide-cellulose conjugate from the reaction mixture. Centrifugation was found to be the more preferred method based on recovery efficiency. The peptide linked conjugate was dried in air and stored at $\sim 4-8{ }^{\circ} \mathrm{C}$ until further use.

ESI-LC/MS Upon the completion of drying, $10 \mathrm{mg}$ of each biosensor was cleaved by adding a cleavage cocktail of TFA/water/triisopropylsilane (95/2.5/2.5) to the biosensor for three hours. The solution for each biosensor was diluted with water (1:10) and submitted for ESI-LC/MS. The generation of the biosensors was confirmed via their molecular weight, which is reported in Table 2.

\section{Fmoc loading substitution}

The supernatant from the deblock solution was measured using UV-VIS spectrophotometer and the loading substitution of the different transducers was calculated (Chan et al. 2000). The supernatant of each matrix was transferred to a quartz cuvette and the absorbance was measure at $301 \mathrm{~nm}$. The loading substitution was calculated using Eq. 1.

Loading $\left(\frac{\mathrm{mmol}}{\mathrm{g}}\right)=\frac{\text { Abs } s_{\text {sample }}}{\text { mg of sample } * 1.75}$

\section{Elemental analysis}

All samples were submitted as solids to Midwest Microlabs (Indianapolis, IN) for carbon (C), hydrogen $(\mathrm{H})$, and nitrogen $(\mathrm{N})$ analysis. Nitrogen content calculations for peptide mass $(\mu \mathrm{g})$ and peptide $(\mu \mathrm{g} /$ mg) were performed in Excel 2007 using Eqs. 2 and 3. The peptide mass determines the amount of peptide on the surface of the biosensor by multiplying the nitrogen mass by the nitrogen factor of 6.25, Eq. 2 . The amount of peptide $(\mu \mathrm{g})$ per $\mathrm{mg}$ of support was achieved by dividing the peptide mass by the weight of the biosensor, Eq. 3. 
Peptide $\operatorname{mass}(\mu g)=N_{\text {mass }}(\mu g) * 6.25$

Peptide $\left(\frac{\mu g}{m g}\right)=\frac{\text { peptide mass }}{\text { weight of biosensor }}$

\section{$X$-ray diffraction $(X R D)$}

All samples were submitted to University of New Orleans Chemistry Department X-Ray diffraction facilities where the tNFC sample was mounted on glass slides with a double-sided tape. The matrices were scanned in the $\theta-2 \theta$ reflection mode with a Philips X'pert powder diffractometer, a $\mathrm{Cu}$ tube, and a graphite monochromator. The data were obtained in a $2 \theta$ scale from $5^{\circ}$ to $40^{\circ}$ with a step size of 0.05 at $2.5 \mathrm{~s}$ per step with an overall runtime of 30 min scans. No background corrections were made. The crystallinity indices $(\mathrm{CrI})$ of the matrices were determined by the minimum intensity near $18^{\circ} 2 \theta\left(\mathrm{I}_{\mathrm{AM}}\right)$ and the maximum intensity (near $22.65^{\circ} 2 \theta, \mathrm{I}_{200}$ ) as shown in Eq. 4 (Segal et al. 1959).

$C r I=\frac{I_{200}-I_{A M}}{I_{200}} \times 100$

Attenuated total reflectance infrared imaging (ATRIR)

A Platinum Alpha ATR-IR Bruker spectrometer, A220/D01 was used to determine the functional groups of the tNFC transducers and biosensors. The OPUS 6.5 software was used to collect sixty-four scans at a resolution of $4 \mathrm{~cm}^{-1}$ in the $4000-500 \mathrm{~cm}^{-1}$ region and to analyze the spectra. All spectra were graphed using Microsoft Excel.

\section{Substitution}

Degree of substitution (DS) of the biosensors was calculated using the Touzinsky and Gordon method where the percent nitrogen was determined using elemental analysis (Touzinsky et al. 1979). The application of $\mathrm{DS}$ herein is as follows: $\mathrm{PC}_{1}$ is the percent nitrogen, $\mathrm{MW}_{\mathrm{u}}$ is the molecular weight of one glucose residue, $\mathrm{MW}_{1}$ is the molecular weight of one nitrogen atom, $\mathrm{N}_{1}$ represents the number of nitrogen atoms, and $\mathrm{MWG}_{1}$ is the molecular weight of the peptide with the PEG linker.
$D S_{1}=\frac{P C_{1} * M W_{u}}{\left[\left(M W_{1}\right)\left(N_{1}\right)(100)-M W G_{1}\right]}$

This value was used in conjunction with the number of chains in each crystallite model to calculate the degree of substitution per crystallite $\left(\mathrm{DS}_{\mathrm{Cr}}\right)$.

\section{Specific surface area by formula}

The specific surface area (SSA) of the tNFC was calculated with a density of $1.6 \mathrm{~g} / \mathrm{cm}^{3}$. The BrunauerEmmett-Teller specific surface area (BET) was determined with a multipoint surface area using $\mathrm{Kr}$ gas adsorption utilizing Micrometrics ASAP 2405 Krypton system (Brunauer et al. 1938). The tNFC sample was first degassed in the Micrometrics ASAP 2405 at $100{ }^{\circ} \mathrm{C}$ for $4 \mathrm{~h}$ prior to the analysis followed by $\mathrm{Kr}$ adsorption at $-196^{\circ} \mathrm{C}$.

\section{Porosity and average pore size}

The porosity of the tNFC material was calculated using Eq. 6 where the density $(\rho)$ of the transducer is divided by the density ( $\left.\rho_{\text {cellulose }}\right)$ of cellulose $(1.46 \mathrm{~g} /$ $\mathrm{cm}^{3}$ ) (Sehaqui et al. 2011). Mercury intrusion analysis measured the porosity and average pore size of the aerogel (0.019-0.061 g) utilizing a Micrometrics AutoPore IV 9500 with a relative pressure ranging from 0.10 to 61,000 psia.

Porosity $=\frac{1-\rho}{\rho_{\text {cellulose }}}$

\section{Crystallite volume and sensors per unit volume}

The volume for each crystallite was based on the volume of a cellulose I $\beta$ crystalline unit cell. The unit cell consists of two cellulose chains, one central chain and four quarter chains at each corner, each with a net of one-half glucose residue. The net total volume is $658 \AA^{3}$. Because the crystallite models were based on a single layer of glucose residues, this volume was divided by two yielding a half unit cell volume of $329 \AA^{3}$. This volume multiplied by the number of chains in each crystallite mode and divided by two results in the crystallite volume. The volume ratio $\left(V_{R}\right)$ is defined as 
$V_{R}=\frac{V_{F P}}{V_{S}}$

where $V_{S}$ is the volume of a given sample crystallite and $\mathrm{V}_{\mathrm{FP}}$ is the volume of the filter paper (FP) sample which occupies the largest volume of all the materials tested and was used as the standard unit for comparison. The result is the number of sample crystallites which can occupy the same space as the FP crystallite. The number of sensors per unit volume was calculated using the volume ratio and degree of substitution per crystallite, $\mathrm{DS}_{\mathrm{Cr}}$.

Sensors per Volume $=D S_{C r} * V_{R}$

\section{Moisture absorption}

The moisture absorption of the tNFC transducer was measured at $0,15,30,60,90,120$, and 180 min after soaking in Millipore water. The sample was weighed, soaked in Millipore water, and removed after each time interval, slightly patted dry with a Kimwipe, and weighed. The swelling capacity of the transducers was determined using Eq. (9) where Ws is the weight of the sample with absorbed water and $\mathrm{Wd}$ is the weight of dry sample.

Swelling $(\%)=\frac{\left(W_{S}-W_{D}\right)}{W_{D}} * 100$

\section{Results and discussion}

Synthesis of biosensors

The transducer surface was generated through the well-known TEMPO-mediated oxidation process (Isogai et al. 2011; Saito et al. 2006, 2007) resulting in the conversion of the glucan C6 and attached primary hydroxyl unit into a carboxylic acid. TEMPOmediated oxidation is regioselective, occurring on the surface at the primary hydroxyl groups and avoids the secondary hydroxyl groups (de Nooy et al. 1994). Moreover, due to the inherent twofold screw axis of cellulose chains, TEMPO-mediated oxidation only affects approximately half of the glucan C6 groups as the other C6 groups are buried within the bulk cellulose material (Habibi et al. 2010). The tNFC sample is a hydrogel-like material due to its hydrophilic nature and is composed of long, thin fibrils. These structures are typically only a few $\mathrm{nm}$ in width and $>1 \mu \mathrm{m}$ in length, providing a large surface area-to-volume ratio (De France et al. 2017).

Characterization of the atomic weight percent of the transducer surface as provided in Table 1 demonstrates the similarity of the experimental and predicted values of the transducer surface consistent with conversion from native cellulose to TEMPO-oxidized cellulose. The predicted values are based on a model molecular structure of a TEMPO-oxidized cellobiose residue in which one glucan ring is TEMPO-oxidized while the other remains in the native state $\left(\mathrm{C}_{12} \mathrm{H}_{18} \mathrm{O}_{11}\right)$, a function of the twofold screw axis (Habibi et al. 2010).

The fluorogenic elastase substrate was attached to the TEMPO-oxidized cellulose surface with a PEG linker as shown in scheme 1. As illustrated, the linker comprising three PEG units was initially attached to the carboxyl of the tNFC, followed by removal of the Fmoc protecting group. The result of this step was denoted tNFC-Peg. The final synthesis step attached the fluorogenic enzyme substrate which consists of a succinyl group, tripeptide sequence Ala-Pro-Ala (APA), and fluorescent 7-amino-4-methylcoumarin (AMC) species. The resulting material was termed tNFC-Pep.

\section{Characterization of biosensors}

The tNFC-Peg and tNFC-Pep samples were analyzed to determine the amount of nitrogen atoms present using electrospray ionization and liquid chromatography-mass spectrometry (ESI-LC/MS). The number of peptides per mg of biosensor was calculated using Eqs. 2 and 3 (see Materials and Methods). The average nitrogen percent, determined through ESILC/MS, is calculated via the Touzinsky and Gordon

Table 1 Carbon, hydrogen, and oxygen weight percent contents of tNFC

\begin{tabular}{llll}
\hline tNFC & $\% \mathrm{C}$ & $\% \mathrm{H}$ & $\% \mathrm{O}$ \\
\hline Experimental $^{2}$ & 43.02 & 6.57 & 50.13 \\
Predicted $^{\mathrm{a}}$ & 42.61 & 5.36 & 52.03 \\
\hline
\end{tabular}

${ }^{\text {a Predicted weight percent based on TEMPO-oxidized structure }}$ of cellobiose $\left(\mathrm{C}_{12} \mathrm{H}_{18} \mathrm{O}_{11}\right)$ 


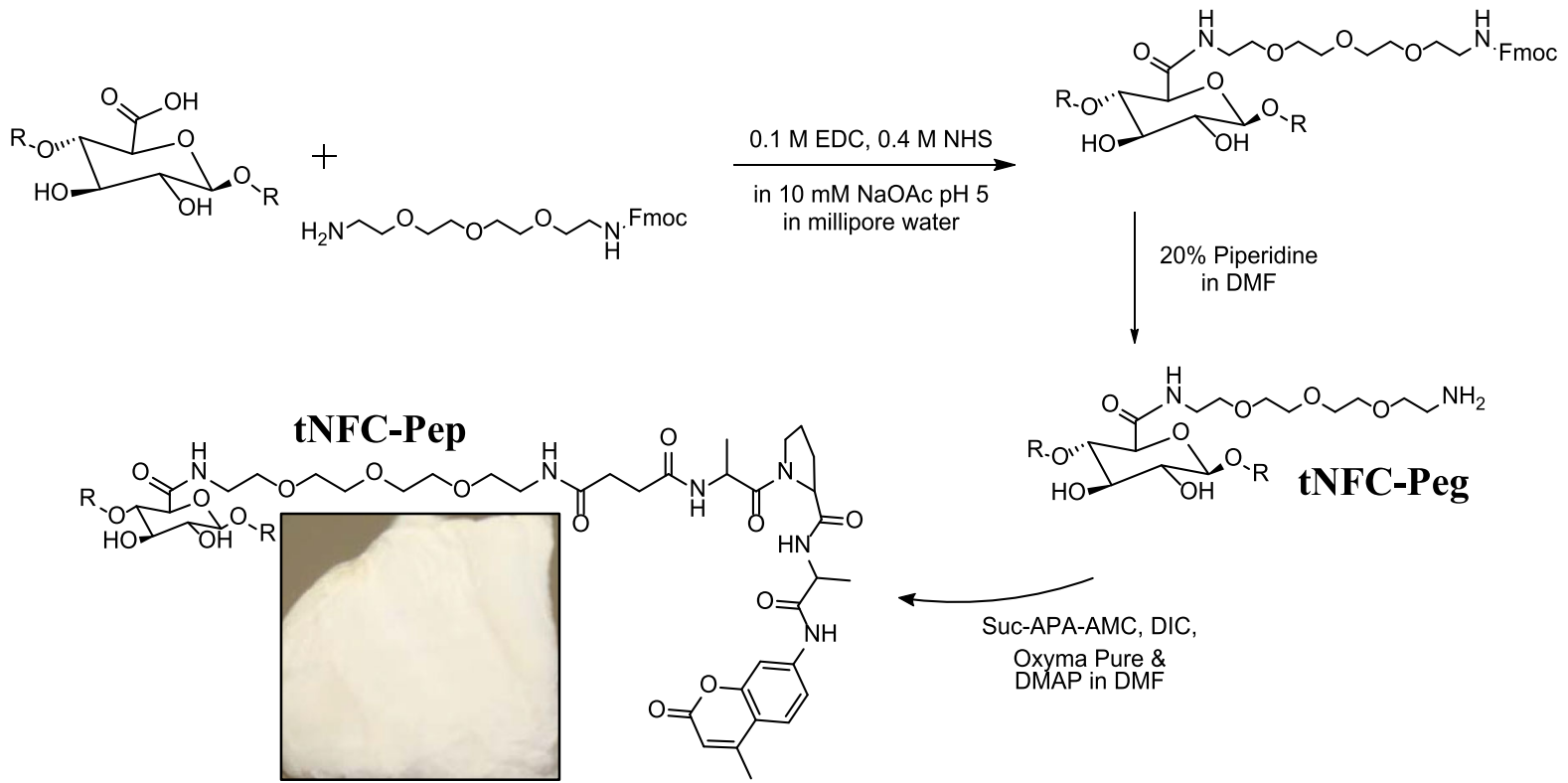

Scheme 1 Addition of the PEG linker and conjugation of the fluorogenic elastase substrate to the tNFC material. The "R" groups represent a continuation of the cellulose chains. The

method (Touzinsky et al. 1979) which has been used to determine peptide-cellulose incorporation (Edwards et al. 2013b; Missoum et al. 2012; Sehaqui et al. 2014). The calculation for the Degree of Substitution (DS) per glucose residue is explained in the Materials and Methods section (Eq. 4).

The values recorded for the elemental analysis and DS calculations are presented in Table 2 for tNFC and contrasted with cellulose-based analogs from previous studies to provide a baseline for sample structure and sensor performance (Edwards et al. 2016a,b). Previously, wood-based cellulose nanocrystals (wCNC) and nanocellulose composites (wNCC), cotton nanocellulosic aerogel (NA), and cotton-based print cloth (PC) fabric and filter paper (FP) were reported as transducer surfaces for protease sensors. Note, the wNCC samples are composed of a mixture of nanoand microcrystalline cellulose which is denoted in the parentheses for each entry, respectively. The previous materials used glycine instead of a PEG linker and are abbreviated with Gly.

The peptide content for tNFC-Pep was determined to be $10.75 \mu \mathrm{g} / \mathrm{mg}$, falling within the range of the other materials, $7-88 \mu \mathrm{g} / \mathrm{mg}$. The DS was calculated for tNFC-Peg and tNFC-Pep based on Eq. 5. There is an expected decrease in DS upon addition of the
tNFC-Peg and tNFC-Pep steps in the synthesis are explicitly labelled. The inset image provides an example of the finished product

tripeptide which is similar to previous studies using the glycine linker (Edwards et al. 2016a, 2016b). This suggests that not all PEG linkers are converted to a full biosensor unit, and approximately $2 / 3$ of the linkers remain unreacted after the final synthesis step. However, the DS values for tNFC-Pep (0.057) are consistent with previous work employing cellulose and nanocomposites (0.005-0.061) (Edwards et al. 2016a).

$\mathrm{X}$-ray crystallography characterization

Figure 1 provides the X-ray diffraction (XRD) pattern of the tNFC sample. This pattern shows the main composition of the TEMPO-oxidized transducer surface is cellulose I $\beta$ by the presence of the strong peak at $22.6^{\circ}((200)$ crystal plane, solid line $)$ and the smaller peak at $16.7^{\circ}$ which represents the convoluted (1-10) and (110) crystal planes (dashed line) (Nam et al. 2016). The crystallinity index (CrI) for tNFC was calculated to be $32 \%$ (Eq. 4). This result is far lower than the $\mathrm{CrI}$ values obtained for most cellulose-based biosensor devices which had CrI values around $80 \%$ (Edwards et al. 2016a). However, such analyses to determine the exact crystallinity values are often difficult because of the presence of amorphous 
Table 2 The ESI-LC/MS, average nitrogen percent and peptide content as determined by elemental analysis of the biosensors

\begin{tabular}{|c|c|c|c|c|c|}
\hline $\mathrm{Name}^{\mathrm{a}}$ & $\mathrm{ESI}^{-L C} / \mathrm{MS}^{\mathrm{b}}(\mathrm{m} / \mathrm{z})$ & Average N\% & Peptide $(\mu \mathrm{g} / \mathrm{mg})$ & $\mathrm{DS}^{\mathrm{c}}$ & Reference \\
\hline tNFC-Peg & & 2.18 & & 0.178 & This study \\
\hline tNFC-Pep & {$[\mathrm{M}+1] 689.35$} & 2.36 & 10.75 & 0.057 & This study \\
\hline wCNC-Gly & {$[\mathrm{M}+1] 671.30$} & 2.17 & 88.13 & 0.276 & Edwards et al. (2016a) \\
\hline wCNC-Pep & & 2.54 & & 0.061 & Edwards et al. (2016a) \\
\hline wNCC-Gly $(66 / 33)^{\mathrm{d}}$ & {$[\mathrm{M}+1] 671.29$} & 0.26 & 60.63 & 0.030 & Edwards et (al. 2016a) \\
\hline wNCC-Pep $(66 / 33)^{\mathrm{d}}$ & & 1.23 & & 0.026 & Edwards et al. (2016a) \\
\hline wNCC-Gly $(50 / 50)^{\mathrm{d}}$ & {$[\mathrm{M}+1] 671.31$} & 0.26 & 30.00 & 0.030 & Edwards et al. (2016a) \\
\hline wNCC-Pep $(50 / 50)^{\mathrm{d}}$ & & 0.74 & & 0.015 & Edwards et al. (2016a) \\
\hline NA-Gly & & 0.43 & & 0.051 & Edwards et al. (2016b) \\
\hline NA-Pep & {$[\mathrm{M}+1] 572.37$} & 0.76 & 20.31 & 0.015 & Edwards et al. (2016b) \\
\hline PC-Gly & [M] 670.33 & 0.12 & 7.19 & 0.014 & Edwards et al. (2016a) \\
\hline PC-Pep & & 0.23 & & 0.005 & Edwards et al. (2016a) \\
\hline FP-Gly & {$[M+1] 671.30$} & 0.19 & 25.00 & 0.022 & Edwards et al. (2016a) \\
\hline FP-Pep & & 0.59 & & 0.012 & Edwards et al. (2016a) \\
\hline
\end{tabular}

${ }^{\mathrm{a}}$ The abbreviation Peg and Gly indicate the linker for each material. Pep indicates the elastase substrate with linker (Peg or Gly) ${ }^{b}[\mathrm{M}+1]$ calculated for tNFC-Pep is based on the full biosensor $\left(\mathrm{C}_{33} \mathrm{H}_{49} \mathrm{~N}_{6} \mathrm{O}_{10}\right)$ with $\mathrm{m} / \mathrm{z} 689.35$ and matches the experimental value ${ }^{c} \mathrm{DS}$ is the degree of substitution as calculated by Touzinsky and Gordon method

${ }^{\mathrm{d}}$ The NCC materials were composed of a mixture of nanocrystalline and microcrystalline cellulose in the ratio provided after each entry, respectively

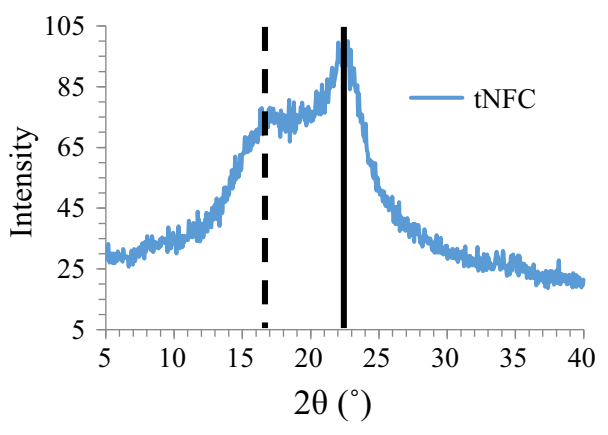

Fig. 1 X-ray diffraction pattern of the tNFC material showing the significant peaks. The vertical lines highlight the peaks of interest for quantifying the results, presenting the (200) crystal plane at $22.6^{\circ}$ (solid line) and (1-10)/(110) crystal planes at $16.7^{\circ}$ (dashed line)

component in tNFC materials. Simulated diffraction patterns suggest the degree of crystallinity could be as high as $\sim 69 \%$ based on estimated values for the (200) crystal plane full-width half max. This amorphous component appears as a broad band from $15^{\circ}$ to $25^{\circ}(2 \theta)$ and significantly overlaps and distorts the peaks necessary for determining crystallinity (Edwards et al. 2018a). Therefore, the calculated crystallinity index should not be taken as a precise value but merely indication that tNFC is composed of cellulose I $\beta$ and amorphous components. Similarly, nanocellulosic aerogel (NA) was previously shown to contain multiple components and adopt a 3-D structure (Edwards et al. 2018a) which suggests the structure of tNFC is more similar to NA than other cellulose-based materials. However, unlike NA, the tNFC pattern lacks a small peak at $12^{\circ}$, which implies there is not a cellulose II component.

The tNFC crystallite sizes were determined through the XRD data by the Scherrer method (Scherrer 1912), and the results of the analysis for the transducer surface with and without attached biosensor are provided in Table 3 along with materials from prior studies (Edwards et al. 2018a; Fontenot et al. 2016). Crystallite sizes were calculated as previously reported for cellulose-based transducer surfaces (Edwards et al. 2016a, 2018a). As presented in the table, the tNFC crystallite sizes with and without the biosensor are essentially the same and compared to the other cellulose- and nanocellulose-based materials, tNFC is slightly smaller. Crystallite models are 
Table 3 The crystallite size, model layers and chain values for each material

\begin{tabular}{llll}
\hline Name & Crystallite Size $^{\mathrm{a}}(\AA)$ & Model (layers/chains) $\left.^{(}\right)$ & Reference \\
\hline tNFC & 25.0 & $6 / 24$ & This study \\
tNFC-Pep & 26.0 & $7 / 30$ & This study \\
wCNC-Pep & 36.0 & $9 / 43$ & Fontenot et al. (2016) \\
wNCC-Pep (66/33) & 32.2 & $8 / 36$ & Fontenot et al. (2016) \\
wNCC-Pep (50/50) & 35.3 & $9 / 43$ & Fontenot et al. (2016) \\
NA-Pep & 58.5 & $12 / 78$ & Edwards et al. (2018a) \\
PC-Pep & 56.3 & $15 / 109$ & Fontenot et al. (2016) \\
FP-Pep & 69.3 & $18 / 144$ & Fontenot et al. (2016), Edwards et al. (2018a)
\end{tabular}

${ }^{\text {a }}$ The crystallite size was determined using the Scherrer formula

illustrated in Fig. 2; Fig. 2a presents the basic tNFC crystallite before undergoing linker attachment, and 2B, C provide the tNFC crystallites with attached peptide on either the (110) crystal plane (2B) or the (1-10) crystal plane (2C). Using the DS values from Table 2, the number of attached biosensor units for tNFC-Pep was calculated at 1.7 for a crystallite of this size. For simplicity of illustration, one sensor was attached to each crystallite model.

Infrared structural analysis

The ATR-IR spectral characterization of the tNFC samples with and without the anchored biosensor is
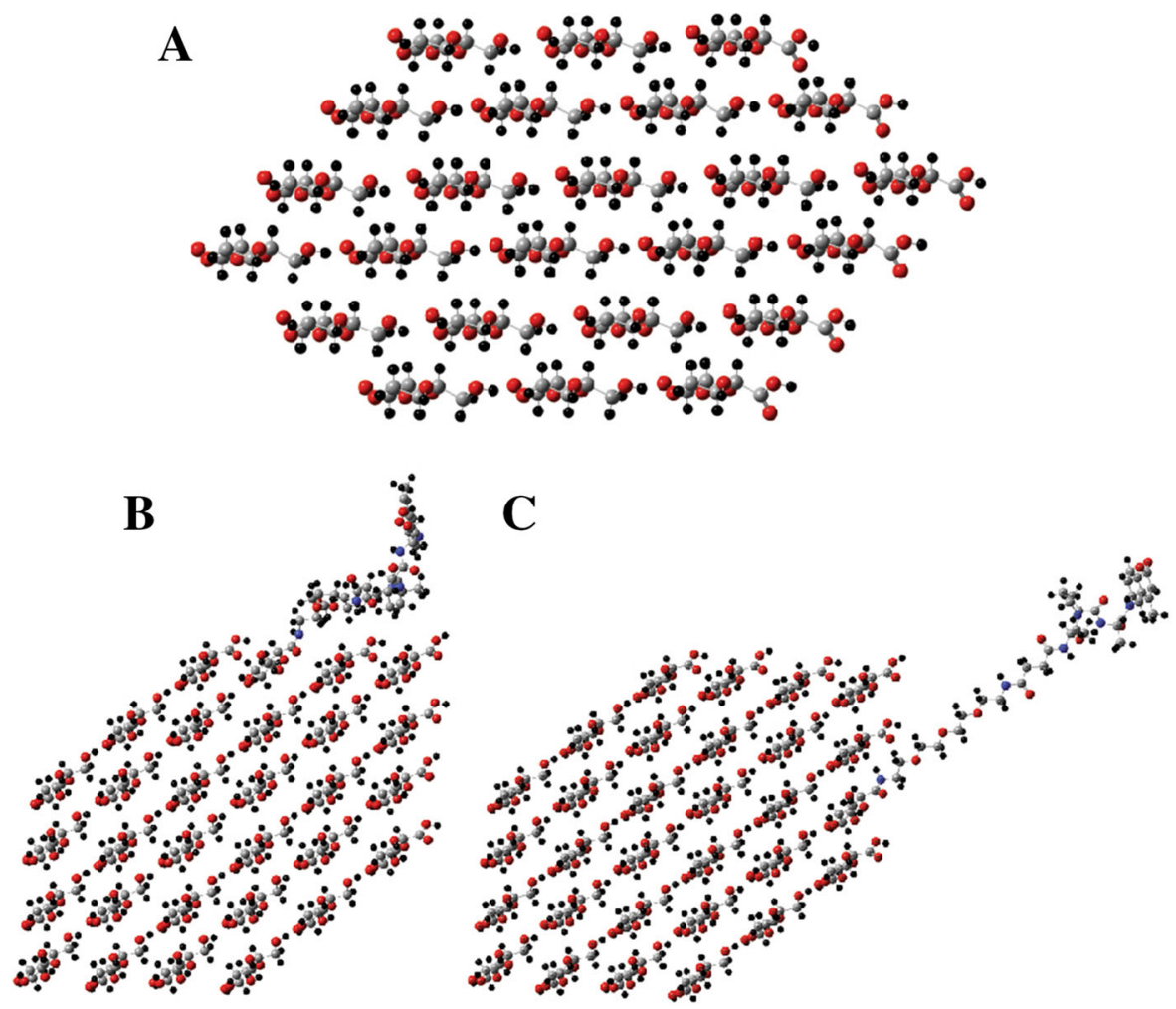

Fig. 2 Model crystallites of tNFC for the a tNFC transducer surface, $\mathbf{b}$ tNFC-Pep with the linker attached to the 110 crystal surface, and c tNFC-Pep with the linker attached to the 1-10 crystal surface 
provided in Fig. 3. As can be seen from the spectra, the line shape shifts from a single Gaussian-shaped peak around $1730 \mathrm{~cm}^{-1}$ (tNFC, 3A), a feature which is indicative of $\mathrm{C}=\mathrm{O}$ stretching vibration of the resulting carboxylic acids from the TEMPO-mediated oxidation process (Habibi et al. 2006; Tang et al. 2017), to a much broader feature with an apparent bimodality containing peaks at 1550 and $1650 \mathrm{~cm}^{-1}$ (tNFC-Pep, 3B). These are highlighted via black arrows in the figure. The spectral change occurs due to the chemical addition of the biosensor unit which converts the carboxyl group of the TEMPO-oxidized transducer surface into an amide group (Scheme 1). These spectral intensities are consistent with previous infrared investigations of TEMPO-oxidized cellulose which show that an amide group anchored to the cellulose surface would produce a broad and weak peak around $1540 \mathrm{~cm}^{-1}$ and a stronger peak near $1650 \mathrm{~cm}^{-1}$ (Orlandin et al. 2014). It should be noted the stronger peak adjacent to the $\mathrm{C}=\mathrm{O}$ mode in Fig. $3 \mathrm{a}$ $\left(1610 \mathrm{~cm}^{-1}\right)$ is attributed to unmodified cellulose. This peak is expected to be more intense as it represents vibrations stemming from the bulk of the cellulose material while the TEMPO-oxidation process only affects the transducer surface. Based on the model crystallites in Fig. 2, only half the surface residues would be oxidized while the other residues (both on the surface and in the bulk) would remain in the native state resulting in a native cellulose: TEMPO cellulose ratio of 3.75. The ratio of the absorbance intensities confirms this as the peak at $1610 \mathrm{~cm}^{-1}$ is nearly fourfold larger than the $\mathrm{C}=\mathrm{O}$ peak at $1730 \mathrm{~cm}^{-1}$.
Calculation of crystallite volume to assess sensor efficacy

Based on the crystallite size (Table 3) and the degree of substitution (Table 2) for each material, the number of biosensors per given volume can be estimated using Eqs. 7 and 8. This is a useful metric to gauge the effectiveness of the sensor signal and sensitivity detection system. As shown in Table 4, tNFC yields the largest crystallite volume ratio as the smallest of the crystallites, nearly a five-fold larger volume ratio than FP, implying that a larger number of tNFC crystallites can fit within the given volume. DS values from Table 2 were employed to determine the number of biosensors per crystallite. The range of DS per Crystallite (Table 4) was from 0.55 to 2.72 , corresponding to the average number of biosensors attached to a model crystallite (Table 3). The results of Eq. 8, utilizing the DS per Crystallite and Volume Ratio, provided the Sensors per Volume for each crystallite material which can be ordered from most to least number of sensors: $w C N C>$ tNFC $>$ wNCC $(66 /$ $33)>\mathrm{NA} \sim \operatorname{wNCC}(50 / 50)>\mathrm{FP}>\mathrm{PC}$.

Additionally, the specific surface area (SSA), material density, porosity, and the average pore size were measured for tNFC. These are presented in Table 5 with materials from previous studies for comparison (Edwards et al. 2016b, 2018b; Fontenot et al. 2016). The results suggest the properties of tNFC are more like nanocellulose aerogels, which supports the findings of the ATR-IR measurements. The porosity and average pore sizes are essentially the same, and while the SSA for tNFC is smaller than NA, the crystallite volume (Table 4) is also smaller; suggesting a larger amount of tNFC crystallites and increased number of sensors would fit within the same volume. While the SSA measurements were

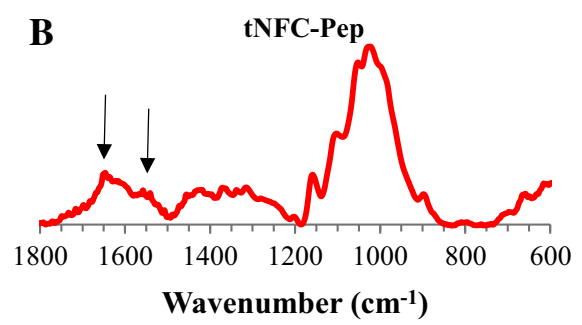

Fig. 3 ATR-IR spectra for a tNFC and b tNFC, PEG linked peptide substrate conjugate with black arrows pointing to the notable spectral changes illustrating the conversion from a carboxyl group (a) to an amide (b) 
Table 4 Calculated crystallite volume for each cellulose-peptide conjugate and number of sensors per unit volume for each material

\begin{tabular}{lclll}
\hline Name & Crystallite volume $^{\mathrm{a}}\left(\mathrm{nm}^{3}\right)$ & Volume ratio $^{\mathrm{b}}$ & DS per crystallite & Sensors per volume $^{\mathrm{c}}$ \\
\hline tNFC & & & & 1.71 \\
tNFC-Pep & 4.94 & 4.80 & 2.62 & 8.21 \\
wCNC-Pep & 7.08 & 3.35 & 0.94 & 3.78 \\
wNCC-Pep (66/33) & 5.93 & 4.00 & 0.64 & 2.14 \\
wNCC-Pep (50/50) & 7.08 & 3.35 & 1.17 & 2.16 \\
NA-Pep & 12.84 & 1.85 & 0.55 & 0.72 \\
PC-Pep & 17.94 & 1.32 & $1.73^{\mathrm{e}}$ & $1.73^{\mathrm{e}}$ \\
FP-Pep & 23.70 & 1.00 & \\
\hline
\end{tabular}

${ }^{\text {a }}$ Crystallite volume was calculated using the known volume of two glucan units and the number of model chains

${ }^{b}$ Volume ratio was calculated as the crystallite volume of the sample compared to FP volume

${ }^{\mathrm{c}}$ DS per crystallite determined by multiplying DS of sample by number of model chains

${ }^{\mathrm{d}}$ Sensors per volume are a function of volume ratio and DS per crystallite

${ }^{\mathrm{e}}$ Values are the same because the FP crystallite was used as the standard unit volume

Table 5 Specific surface area, density, porosity, and average pore size values obtained for the materials

\begin{tabular}{|c|c|c|c|c|c|}
\hline Name & $\begin{array}{l}\mathrm{SSA}^{\mathrm{a}}\left(\mathrm{m}^{2} /\right. \\
\mathrm{g})\end{array}$ & $\begin{array}{l}\text { Density } \\
\mathrm{g} / \mathrm{cm}^{3}\end{array}$ & $\begin{array}{l}\text { Porosity } \\
(\%)\end{array}$ & $\begin{array}{l}\text { Average Pore Size } \\
(\mu \mathrm{m})\end{array}$ & Reference \\
\hline $\mathrm{tNFC}$ & 27.57 & 0.011 & 99 & 0.012 & This study \\
\hline wCNC & 261.2 & 1.600 & - & - & Fontenot et al. (2016) \\
\hline $\begin{array}{l}\text { wNCC }(66 / \\
33)\end{array}$ & 0.021 & 0.290 & 28.65 & 0.048 & Fontenot et al. (2016) \\
\hline $\begin{array}{l}\text { wNCC }(50 / \\
50)\end{array}$ & 0.035 & 0.270 & 24.54 & 0.033 & Fontenot et al. (2016) \\
\hline NA & 162.94 & - & 98.9 & 0.011 & Edwards et al. (2016b, 2018b) \\
\hline $\mathrm{PC}$ & 0.016 & 0.613 & 58.01 & - & Fontenot et al. (2016) \\
\hline FP & 0.020 & 0.502 & 65.62 & $20-25$ & $\begin{array}{l}\text { Fontenot et al. (2016), Edwards et al. } \\
(2018 b)\end{array}$ \\
\hline
\end{tabular}

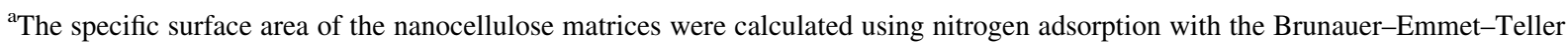
theory

${ }^{\mathrm{b}}$ The porosity for the tNFC foam was estimated based on Eq. 6 . The average pore size for all nanocellulosic materials was calculated using nitrogen adsorption with the Brunauer-Emmet-Teller theory

performed on dry samples, tNFC- and NA-derived hydrogel-like materials swell considerably upon hydration further increasing their SSA. This swelling is illustrated in Fig. 4 which presents a moisture absorption study for the tNFC material over a $3 \mathrm{~h}$ period. The hydrogel-like nature of the tNFC material allows it to absorb a considerable amount of water and swell to nearly $10,000 \%$ its initial volume. Comparing this to materials measured previously, including PC,
NA, and NCC (Fontenot et al. 2017), the moisture uptake is significantly higher for tNFC.

\section{Conclusions}

TEMPO-oxidized nanofibrillated cellulose was prepared as a transducer surface to be utilized as an anchoring material in a biosensor system for the 


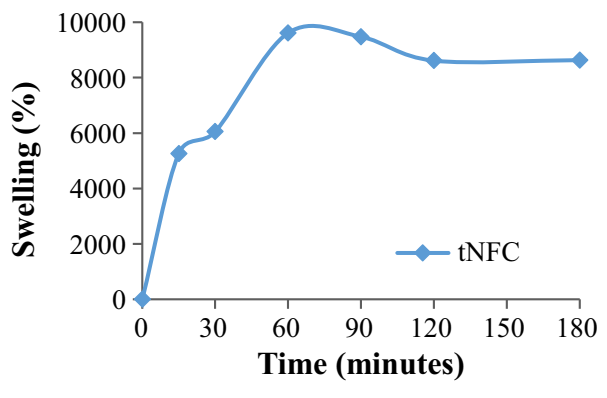

Fig. 4 Moisture absorption studies of the tNFC transducer over a $3 \mathrm{~h}$ period at room temperature

purpose of detecting elevated levels of serine proteases in chronic wounds. The characterization of the sample showed the crystalline component of the material is cellulose I $\beta$, yet tNFC contains a substantial amorphous component according to XRD measurements (32\% crystalline, $68 \%$ amorphous). The crystallite size is comparatively smaller than other cellulose-based materials, which leads to a relatively large SSA and crystallite volume ratio. The characterization techniques including ESI-LC/MS, elemental analysis, and infrared studies confirm the attachment of the peptide-based biosensor with PEG linker to the transducer surface. The degree of substitution for the peptide incorporation into the nanocellulose material was within the range expected based on previously studied samples. However, further analysis on the crystallite volume and DS per crystallite suggests tNFC has one of the highest number of sensors per crystallite compared to the other materials. Coupling this property with the hydrogellike nature of the material (i.e. ability to swell and further increase its SSA), tNFC would provide greater access to HNE and thus more sensitive to detecting elevated levels of the protease in chronic wounds. The synthesis and characterization of this TEMPO-oxidized material provides the basis for the development and optimization of novel POC diagnostic devices.

Funding Research was funded through support by the United States Department of Agriculture.

\section{Declarations}

Conflict of interest The authors declare no conflicts of interest that are relevant to this article.

Ethical approval This study does not contain any human or animal participants. All authors state they adhere to the Ethical
Responsibilities of Authors and that the work complies with ethical standards.

Open Access This article is licensed under a Creative Commons Attribution 4.0 International License, which permits use, sharing, adaptation, distribution and reproduction in any medium or format, as long as you give appropriate credit to the original author(s) and the source, provide a link to the Creative Commons licence, and indicate if changes were made. The images or other third party material in this article are included in the article's Creative Commons licence, unless indicated otherwise in a credit line to the material. If material is not included in the article's Creative Commons licence and your intended use is not permitted by statutory regulation or exceeds the permitted use, you will need to obtain permission directly from the copyright holder. To view a copy of this licence, visit http://creativecommons.org/licenses/by/4.0/.

\section{References}

Blackwell HE (2006) Hitting the spot: small-molecule macroarrays advance combinatorial synthesis. Curr Opin Chem Biol 10:203-212. https://doi.org/10.1016/j.cbpa. 2006.04.026

Brunauer S, Emmett PH, Teller E (1938) Adsorption of gases in multimolecular layers. J Am Chem Soc 60:309-319. https://doi.org/10.1021/ja01269a023

Calahorra Y, Datta A, Famelton J, Kam D, Shoseyov O, KarNarayan S (2018) Nanoscale electromechanical properties of template-assisted hierarchical self-assembled cellulose nanofibers. Nanoscale 10:16812-16821. https://doi.org/10. 1039/C8NR04967J

Chan WC, White PD (eds) (2000) Fmoc solid phase peptide synthesis: a practical approach. Oxford University Press, p 376. https://doi.org/10.1093/oso/9780199637256.001. 0001

Chen J, Nugen SR (2019) Detection of protease and engineered phage-infected bacteria using peptide-graphene oxide nanosensors. Anal Bioanal Chem 411:2487-2492. https:// doi.org/10.1007/s00216-019-01766-6

Choi S, Goryll M, Sin LYM, Wong PK, Chae J (2011) Microfluidic-based biosensors toward point-of-care detection of nucleic acids and proteins. Microfluid Nanofluid 10:231-247. https://doi.org/10.1007/s10404-0100638-8

Courtenay JC, Sharma RI, Scott JL (2018) Recent advances in modified cellulose for tissue culture applications. Molecules 23:654. https://doi.org/10.3390/molecules23030654

De France KJ, Hoare T, Cranston ED (2017) Review of hydrogels and aerogels containing nanocellulose. Chem Mater 29:4609-4631. https://doi.org/10.1021/acs. chemmater.7b00531

de Nooy AEJ, Besemer AC, Hv B (1994) Highly selective tempo mediated oxidation of primary alcohol groups in polysaccharides. Recl Trav Chim Pays-Bas 113:165-166. https://doi.org/10.1002/recl.19941130307

Doench I, Ahn Tran T, David L, Montembault A, Viguier E, Gorzelanny C, Sudre G, Cachon T, Louback-Mohamed M, Horbelt N, Peniche-Covas C, Osorio-Madrazo A (2019) 
Cellulose nanofiber-reinforced chitosan hydrogel composites for intervertebral disc tissue repair. Biomimetics (Basel). https://doi.org/10.3390/biomimetics4010019

Edwards JV, Prevost N, French A, Concha M, DeLucca A, Wu Q (2013a) Nanocellulose-based biosensors: design, preparation, and activity of peptide-linked cotton cellulose nanocrystals having fluorimetric and colorimetric elastase detection sensitivity. Engineering 5:20-28. https://doi.org/ 10.4236/eng.2013.59A003

Edwards JV, Prevost N, Sethumadhavan K, Ullah A, Condon B (2013b) Peptide conjugated cellulose nanocrystals with sensitive human neutrophil elastase sensor activity. Cellulose 20:1223-1235. https://doi.org/10.1007/s10570-0139901-y

Edwards JV, Fontenot KR, Haldane D, Prevost NT, Condon BD, Grimm C (2016a) Human neutrophil elastase peptide sensors conjugated to cellulosic and nanocellulosic materials: part I, synthesis and characterization of fluorescent analogs. Cellulose 23:1283-1295. https://doi.org/10.1007/ s10570-016-0869-2

Edwards JV, Fontenot KR, Prevost NT, Pircher N, Liebner F, Condon BD (2016b) Preparation, characterization and activity of a peptide-cellulosic aerogel protease sensor from cotton. Sensors 16:1789. https://doi.org/10.3390/ s16111789

Edwards JV, Fontenot K, Liebner F, Pircher NDN, French AD, Condon BD (2018) Structure/function analysis of cottonbased peptide-cellulose conjugates: spatiotemporal/kinetic assessment of protease aerogels compared to nanocrystalline and paper cellulose. Int J Mol Sci 19:840. https:// doi.org/10.3390/ijms19030840

Edwards JV, Fontenot KR, Liebner F, Condon BD (2018b) Peptide-cellulose conjugates on cotton-based materials have protease sensor/sequestrant activity. Sensors 18:2334. https://doi.org/10.3390/s18072334

Fontenot KR, Edwards JV, Haldane D, Graves E, Citron MS, Prevost NT, French AD, Condon BD (2016) Human neutrophil elastase detection with fluorescent peptide sensors conjugated to cellulosic and nanocellulosic materials: part II, structure/function analysis. Cellulose 23:1297-1309. https://doi.org/10.1007/s10570-016-0873-6

Fontenot KR, Edwards JV, Haldane D, Pircher N, Liebner F, Condon BD, Qureshi H, Yager D (2017) Designing cellulosic and nanocellulosic sensors for interface with a protease sequestrant wound-dressing prototype: implications of material selection for dressing and protease sensor design. J Biomater Appl 32:622-637. https://doi.org/10. 1177/0885328217735049

Habibi Y, Chanzy H, Vignon MR (2006) Tempo-mediated surface oxidation of cellulose whiskers. Cellulose 13:679-687. https://doi.org/10.1007/s10570-006-9075-y

Habibi Y, Lucia LA, Rojas OJ (2010) Cellulose nanocrystals: chemistry, self-assembly, and applications. Chem Rev 110:3479-3500. https://doi.org/10.1021/cr900339w

Huang H, Su S, Wu N, Wan H, Wan S, Bi H, Sun L (2019) Graphene-based sensors for human health monitoring. Front Chem 7:399. https://doi.org/10.3389/fchem.2019. 00399

Isogai A, Saito T, Fukuzumi H (2011) Tempo-oxidized cellulose nanofibers. Nanoscale 3:71-85. https://doi.org/10. 1039/C0NR00583E
Jurado-Sánchez B (2018) Nanoscale biosensors based on selfpropelled objects. Biosensors. https://doi.org/10.3390/ bios8030059

Kumar S, Kumar S, Ali MA, Anand P, Agrawal VV, John R, Maji S, Malhotra BD (2013) Microfluidic-integrated biosensors: prospects for point-of-care diagnostics. Biotechnol J 8:1267-1279. https://doi.org/10.1002/biot. 201200386

Le Ferrand H, Chabi S, Agarwala S (2020) 3d assembly of graphene nanomaterials for advanced electronics. Adv Intell Syst 2:1900151. https://doi.org/10.1002/aisy. 201900151

Liu Y, Matharu Z, Howland MC, Revzin A, Simonian AL (2012) Affinity and enzyme-based biosensors: recent advances and emerging applications in cell analysis and point-of-care testing. Anal Bioanal Chem 404:1181-1196. https://doi.org/10.1007/s00216-012-6149-6

Mao R, Yao W, Qadir A, Chen W, Gao W, Xu Y, Hu H (2020) 3-d graphene aerogel sphere-based flexible sensors for healthcare applications. Sens Actuator, A 312:112144. https://doi.org/10.1016/j.sna.2020.112144

Min J, Nothing M, Coble B, Zheng H, Park J, Im H, Weber GF, Castro CM, Swirski FK, Weissleder R, Lee H (2018) Integrated biosensor for rapid and point-of-care sepsis diagnosis. ACS Nano 12:3378-3384. https://doi.org/10. 1021/acsnano.7b08965

Missoum K, Belgacem MN, Barnes J-P, Brochier-Salona M-C, Bras J (2012) Nanofibrillated cellulose surface grafting in ionic liquid. Soft Matter. https://doi.org/10.1039/ C2SM25691F

Mohanty SP, Kougianos E (2006) Biosensors: a tutorial review. IEEE Potentials 25:35-40. https://doi.org/10.1109/MP. 2006.1649009

Nam S, French AD, Condon BD, Concha M (2016) Segal crystallinity index revisited by the simulation of $\mathrm{x}$-ray diffraction patterns of cotton cellulose I $\beta$ and cellulose II. Carbohydr Polym 135:1-9. https://doi.org/10.1016/j. carbpol.2015.08.035

Orlandin A, Formaggio F, Toffoletti A, Peggion C (2014) Cotton functionalized with peptides: characterization and synthetic methods. J Pept Sci 20:547-553. https://doi.org/ 10.1002/psc. 2659

Portela R, Leal CR, Almeida PL, Sobral RG (2019) Bacterial cellulose: a versatile biopolymer for wound dressing applications. Microb Biotechnol 12:586-610. https://doi. org/10.1111/1751-7915.13392

Saito T, Nishiyama Y, Putaux J-L, Vignon M, Isogai A (2006) Homogeneous suspensions of individualized microfibrils from tempo-catalyzed oxidation of native cellulose. Biomacromol 7:1687-1691. https://doi.org/10.1021/ bm060154s

Saito T, Kimura S, Nishiyama Y, Isogai A (2007) Cellulose nanofibers prepared by tempo-mediated oxidation of native cellulose. Biomacromol 8:2485-2491. https://doi.org/10. 1021/bm0703970

Scherrer P (1912) Bestimmung der inneren Struktur und der Größe von Kolloidteilchen mittels Röntgenstrahlen. In: Kolloidchemie Ein Lehrbuch. Chemische Technologie in Einzeldarstellungen. Springer, Berlin, Heidelberg. https:// doi.org/10.1007/978-3-662-33915-2_7 
Schlesinger M, Giese M, Blusch LK, Hamad WY, MacLachlan MJ (2015) Chiral nematic cellulose-gold nanoparticle composites from mesoporous photonic cellulose. Chem Commun 51:530-533. https://doi.org/10.1039/ C4CC07596J

Segal L, Creely JJ, Martin AEJ, Conrad CM (1959) An empirical method for estimating the degree of crystallinity of native cellulose using the $\mathrm{x}$-ray diffractometer. Text Res J 29:786-794. https://doi.org/10.1177/ 004051755902901003

Sehaqui H, Zhou Q, Ikkala O, Berglund LA (2011) Strong and tough cellulose nanopaper with high specific surface area and porosity. Biomacromol 12:3638-3644. https://doi.org/ 10.1021/bm2008907

Sehaqui H, Zimmermann T, Tingaut P (2014) Hydrophobic cellulose nanopaper through a mild esterification procedure. Cellulose 21:367-382. https://doi.org/10.1007/ s10570-013-0110-5

Song W, Lee JK, Gong MS, Heo K, Chung WJ, Lee BY (2018) Cellulose nanocrystal-based colored thin films for colorimetric detection of aldehyde gases. ACS Appl Mater Interfaces 10:10353-10361. https://doi.org/10.1021/ acsami.7b19738

Soper SA, Brown K, Ellington A, Frazier B, Garcia-Manero G, Gau V, Gutman SI, Hayes DF, Korte B, Landers JL, Larson D, Ligler F, Majumdar A, Mascini M, Nolte D, Rosenzweig Z, Wang J, Wilson D (2016) Point-of-care biosensor systems for cancer diagnostics/prognostics. Biosens Bioelectron 21:1932-1942. https://doi.org/10.1016/j.bios. 2006.01.006
Tamayo J, Kosaka PM, Ruz JJ, Pauloa ÁS, Calleja M (2013) Biosensors based on nanomechanical systems. Chem Soc Rev 42:1287-1311. https://doi.org/10.1039/C2CS35293A

Tang Z, Li W, Lin X, Xiao H, Miao Q, Huang L, Chen L, Wu H (2017) Tempo-oxidized cellulose with high degree of oxidation. Polymers 9:421. https://doi.org/10.3390/ polym 9090421

Thévenot DR, Toth K, Durst RA, Wilson GS (1999) Electrochemical biosensors: recommended definitions and classification. Pure Appl Chem 71:2333-2348. https://doi.org/ 10.1016/S0956-5663(01)00115-4

Touzinsky GF, Gordon SH (1979) Degree of substitution of cellulose derivatives containing $\mathrm{n}$ different substituent groups. Carbohyd Res 69:327-329. https://doi.org/10. 1016/S0008-6215(00)85787-0

Vasan ASS, Mahadeo DM, Doraiswami R, Huang Y, Pecht M (2013) Point-of-care biosensor system. Front Biosci (Schol Ed) 5:39-71. https://doi.org/10.2741/s357

Wang J (2006) Electrochemical biosensors: towards point-ofcare cancer diagnostics. Biosens Bioelectron 21:1887-1892. https://doi.org/10.1016/j.bios.2005.10.027

Zhang T, Wang W, Zhang D, Zhang X, Ma Y, Zhou Y, Qi L (2010) Biotemplated synthesis of gold nanoparticle-bacteria cellulose nanofiber nanocomposites and their application in biosensing. Adv Func Mater 20:1152-1160. https://doi.org/10.1002/adfm.200902104

Publisher's Note Springer Nature remains neutral with regard to jurisdictional claims in published maps and institutional affiliations. 